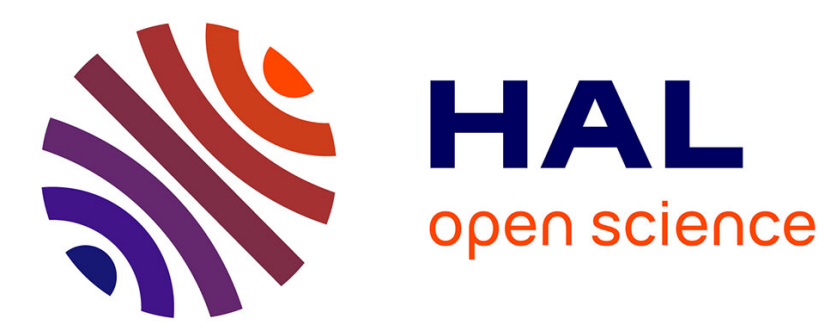

\title{
DEFECT STUDIES OF THIN LAYERS BY THE VIBRATING-REED TECHNIQUE
}

\author{
B. Berry, W. Pritchet
}

\section{To cite this version:}

B. Berry, W. Pritchet. DEFECT STUDIES OF THIN LAYERS BY THE VIBRATINGREED TECHNIQUE. Journal de Physique Colloques, 1981, 42 (C5), pp.C5-1111-C5-1122. 10.1051/jphyscol:19815172 . jpa-00221048

\section{HAL Id: jpa-00221048 https://hal.science/jpa-00221048}

Submitted on 1 Jan 1981

HAL is a multi-disciplinary open access archive for the deposit and dissemination of scientific research documents, whether they are published or not. The documents may come from teaching and research institutions in France or abroad, or from public or private research centers.
L'archive ouverte pluridisciplinaire HAL, est destinée au dépôt et à la diffusion de documents scientifiques de niveau recherche, publiés ou non, émanant des établissements d'enseignement et de recherche français ou étrangers, des laboratoires publics ou privés. 


\title{
DEFECT STUDIES OF THIN LAYERS BY THE VIBRATING-REED TECHNIQUE
}

\author{
B.S. Berry and W.C. Pritchet \\ IBM Thomas J. Watson Research Center, Yorktoun Heights, New York 10598, U.S.A.
}

\begin{abstract}
The vibrating-reed technique has been successfully adapted to internal friction studies of thin-layer materials. Three examples are described, related to the oxygen contamination of niobium films, grain-boundary sliding of aluminum films, and anelasticity due to hydrogen in metallic glasses. Emphasis is given to the use of thin high-Q substrates as a means of examining supported films down to $0.1 \mu \mathrm{m}$ thickness. It has been found that the behavior of films can be radically different from that of bulk samples. For example, two-stage grain-boundary sliding with an activation energy of only $0.55 \mathrm{eV}$ has been observed in aluminum films. In metallic glasses, a peak associated with the presence of hydrogen in small lowsymmetry interstitial sites has been found in many different alloys, including $\mathrm{Pd}_{80} \mathrm{Si}_{20}, \mathrm{Fe}_{80} \mathrm{~B}_{20}, \mathrm{Nb}_{40} \mathrm{Ni}_{60}$ and $\mathrm{Cu}_{57} \mathrm{Zr}_{43}$.
\end{abstract}

1. Introduction.- The wide applicability of internal friction measurements for the study of defects in solids is based upon two circumstances. First, many defects are stress active, as evidenced by such phenomena as point-defect reorientation, dislocation bowing, and grain-boundary sliding. Secondly, internal friction measurements can be applied to all types of solids, regardless of whether they are metallic, semiconducting, insulating, crystalline, or amorphous. Internal friction can thus be regarded as a solid-state tool in the truest sense. It is unnecessary at this conference to extol the virtues of internal friction measurements. Rather, it is our purpose to demonstrate their utility in an area which has so far received comparatively little attention. This is the area of thin-layer materials. One need reflect only briefly on the importance of thin-film technology, or on the high level of activity in the field of metallic glasses, to realize that the province of thin-layer materials encompasses some of the most exciting and technologically-significant areas of present-day materials science.

2. The Vibrating-Reed Technique.- A simple cantilever specimen has a number of uses for thin-layer studies. Perhaps the earliest application involved the static bending of a composite sample, consisting of a thin layer on a supporting substrate, to obtain the sign and magnitude of the internal stress in the layer. For present purposes, we are concerned with the dynamical behavior of a thin cantilever or vibrating reed. Reed samples typically 
possess a convenient set of natural frequencies in the audio range. Their intrinsic flexibility facilitates the excitation of overtones, which is often a feature of significant value.

In some instances, the material of interest may itself provide a vibrating-reed sample without the need for a supporting substrate. However, in the thin-film regime below $1 \mu \mathrm{m}$, the only practical experimental approach involves the use of a substrate. We have learned that such composite samples have several attractive features, and should not be regarded as necessary but inferior alternatives to homogeneous samples. For example, thin detached films of ten have a tendency to curl, which can seriously interfere with their use as vibrating reeds. This problem can be avoided with a composite sample, consisting of nominally identical layers on both sides of a thicker substrate. Of greater importance, we have found that some of the most interesting aspects of the behavior of thin layers in fact relate to their attachment to the substrate, as will become evident in Section 3. There is of course an obvious potential difficulty with the use of a composite sample if the layers of interest, of total thickness $t$, are only a small fraction of the substrate thickness, $d$. This is the problem of the substrate damping, which must be small so that the losses of interest are not masked by substrate losses. In terms of the fractional energy loss per cycle, $\Delta W / W$, the logarithmic decrement $\delta$ of a composite sample may be written

$$
\delta=\Delta W / 2 W=\left(\Delta W_{s}+\Delta W_{\ell}\right) / 2\left(W_{s}+W_{\ell}\right)
$$

where the suffixes $s$ and $\ell$ refer to the substrate and to the layer(s), respectively. If $t / d$ is small enough that $W_{\ell}$ can be neglected in comparison with $W_{s}$, Eq. (1) becomes

$$
\delta=\delta_{s}+\left(W_{\ell} / W_{s}\right) \delta_{\ell}
$$

where $\delta_{s}$ and $\delta_{\ell}$ represent the damping of the substrate and the layer(s), if tested individually under comparable conditions. The observed damping, $\delta$, is seen to be a weighted sum of $\delta_{s}$ and $\delta_{\ell}$, where the weighting factor $W_{\ell} / W_{s}$ is much smaller than unity. Clearly, we then require $\delta_{s}<<\delta_{\ell}$ if the second term in Eq(2) is to contribute significantly to $\delta$. A simple calculation shows the weighting factor may be written

$$
W_{\ell} / W_{s}=(3 t / d)\left(E_{\ell}^{\prime} / E_{s}\right)
$$

where the helpful factor of 3 comes from the advantageous distribution of strain energy over the cross-section of the reed, and $E_{s}$ is Young's modulus of the substrate. To a first approximation, $E^{\prime}{ }_{\ell}$ can be regarded simply as Young's modulus of the detached layer, $E_{\ell}$. To a better approximation, $E_{\ell}^{\prime}$ is a modified Young's modulus, corresponding to the uniaxial stiffness of the layer when constrained to deform with the Poisson's ration $v_{s}$ of the substrate rather than with its own unconstrained value, $\nu_{\ell}$. The relationship between 
$E_{\ell}^{\prime}$ and $E_{\ell}$ is

$$
E_{\ell}^{\prime} / E_{\ell}=\left(1-v_{s} y_{\ell}\right) /\left(1-v_{\ell}^{2}\right)
$$

As an example, for a layer of $\nu_{\ell}=0.33$ on a fused silica substrate $\left(\nu_{s}=0.17\right)$, Eq. (4) gives the result $E^{\prime}{ }_{\ell} / E_{\ell}=1.06$. Fused silica is of particular interest as a substrate because of its low internal friction, which is usually less than $10^{-5}$. With this information, we may quickly assess the ability to detect losses from added surface layers. For example, if films of only $0.1 \mu \mathrm{m}$ thickness are added to each side of a $50 \mu \mathrm{m}$ substrate, and $E_{\ell}^{\prime} \simeq E_{s}$, Eq. (2) becomes, approximately,

$$
\delta \simeq \delta_{s}+0.01 \delta_{\ell}
$$

Thus, if $\delta_{s}<10^{-5}, \delta_{\ell}$ can be detected down to a level of about $10^{-3}$. This provides excellent resolution for many investigations.

3. Illustrative Results.- We describe below three applications of the vibrating-reed technique.[1] The first two make use of composite samples, prepared by vacuum deposition of thin films on $50 \mu \mathrm{m}$ fused silica substrates. The last example is concerned with the hydrogen peak in metallic glasses, and involves the use of self-supporting samples.

\subsection{Oxygen Contamination of Niobium Films}

The Snoek relaxation, associated initially with the interstitial impurities carbon and nitrogen in bcc iron, is undoubtedly the best-known of all point-defect relaxations. This is due in part to the fact that the relaxation was one of the first to be understood in terms of a simple atomic mechanism. Also, of course, a large number of Snoek peaks are now known in other bcc metals. On the other hand, the applications of the Snoek relaxation have also been a substantial factor contributing to its prominence. These applications include the determination of precise diffusion parameters, and the use of the peak as an analytical tool for the amount of interstitial solute dispersed in solid solution. Both of these aspects are of interest in the application of the Snoek relaxation to thin films.

Thin films of the bcc transitional metals are of current interest for a number of reasons, including (i) good substrate adhesion, (ii) use as a diffusion or reaction barrier in multilayer composites, and (iii) use in the formation of a silicide contact on a silicon surface. In addition, individual transitional metals may possess unique properties that make them of special interest for particular applications. For example, niobium has a relatively high superconducting transition temperature $(9 \mathrm{~K})$, and is of interest for the fabrication of thin-film Josephson devices. Niobium is also known as one of the bcc metals with a great tendency to scavenge oxygen, which tends to degrade its superconducting behavior. Fig. 1 illustrates the use of the oxygen Snoek peak to follow progressive contamination of 
niobium films when they are heated in a normal vacuum of 1 to $0.1 \mathrm{mPa}$ to temperatures that allow the diffusion of oxygen to take place. The data were obtained with a composite sample consisting of $0.45 \mu \mathrm{m}$ films on each side of a $50 \mu \mathrm{m}$ silica substrate. The films were deposited in a vacuum of $30 \mu \mathrm{Pa}$ at a rate of $2 \mathrm{~nm} / \mathrm{sec}$, by e-gun evaporation of high-purity $\mathrm{Nb}$ in an ion-pumped oil-free system gettered by a $\mathrm{Ti}$ sublimation pump. The substrate was held at $723 \mathrm{~K}$, and rotated periodically to obtain films on both sides. After transfer to the internal friction apparatus, this sample exhibited on first heating a relatively small peak at the expected location of the oxygen Snoek peak (Fig. 1). A significant enhancement of the peak was already evident during the return run to room temperature. Exposures to progressively higher temperatures are seen to produce further large increases in peak height, and a broadening of the high-temperature side of the peak which is indicative of clustering. A small bump is also evident in the later curves at the expected location of the nitrogen peak. The data of Fig. 1 demonstrate very well the excellent resolution of a relaxation in thin films which together form only $2 \%$ of the thickness of the composite sample. Since the substrate damping is negligible, Fig. 1 gives an immediate relative indication of the buildup of the oxygen content of the films. For the purpose of semiquantitative analysis, the ordinate of Fig. 1 may be multiplied by the factor $(d / 3 t)\left(E_{s} / E_{\ell}^{\prime}\right)$ to obtain the film damping $\delta_{\ell}$ of Eq. (2). The peaks may then be converted to an approximate oxygen content by the use of the calibration factor found from studies of bulk samples. In this way, we estimate that the films initially contained about $0.3 \mathrm{a} / \mathrm{o}$ oxygen in solution. After the first heating to $246^{\circ} \mathrm{C}$, this increased to $0.48 \mathrm{a} / \mathrm{o}$. Since the films

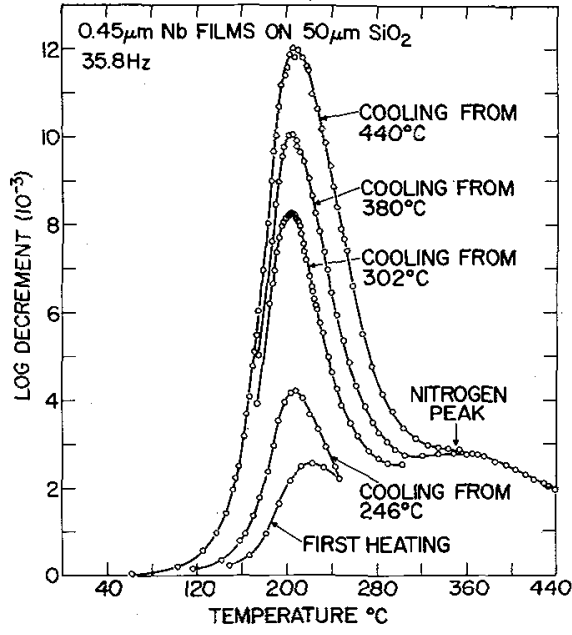

Fig. 1: Growth of the oxygen Snoek peak in thin-film niobium, caused by thermal cycling treatments in vacuum.

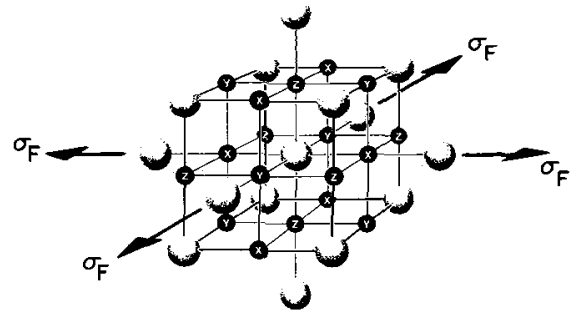

Fig. 2: Schematic illustration of a tensile biaxial film stress $\sigma_{F}$ acting on a $\{100\}$ oriented bcc single-crystal film. The dark circles represent the octahedral interstitial sites, which form 3 subsets of orientations $\mathrm{X}, \mathrm{Y}$ and $\mathrm{Z}$. 
contain about 3500 atomic layers of niobium, the increment of $0.18 \mathrm{a} / \mathrm{o}$ is equivalent to the sorption of about 6 monolayers of oxygen (i.e. 3 monolayers per side). From this viewpoint, the initial sensitivity of the measurements is better than one monolayer of oxygen. At the other extreme, we estimate (with allowance for peak broadening) that, after exposure to $440^{\circ} \mathrm{C}$, the oxygen content of the films has risen an order of magnitude, to $3 \mathrm{a} \%$.

Although the use of the bulk calibration factor is adequate for semi-quantitative analysis, there are at least two reasons why this procedure is not precise. One is the possibility of a different texture in the film. The second reason is related to the very high internal stress which can be present in supported refractory metal films. At room temperature, the biaxial internal stress initially present in the sample of Fig. 1 is known to be $1.5 \mathrm{GPa}$ (tensile), from the curvature exhibited by a sister sample prepared at the same time, but masked on one side. The important effects which a stress of this magnitude can exert on the Snoek relaxation can be most simply discussed with reference to the $\{100\}$ single-crystal film of Fig. 2. Here, it is apparent that a biaxial tension will tend to depopulate the $\mathrm{Z}$-sites, while favoring the $\mathrm{X}$ and $\mathrm{Y}$ sites equally. For, a compressive stress, the situation is reversed. The calculated repopulation, or Snoek ordering, which can occur under the influence of the biaxial stress $\sigma_{F}$ is shown in Fig. 3. The important point is that the internal stresses which can actually occur in thin films are large enough to disturb significantly the relative populations of interstitials in the $X, Y$ and $Z$ sites. As a consequence, the calibration factor $K$ relating the peak height to oxygen content also becomes a

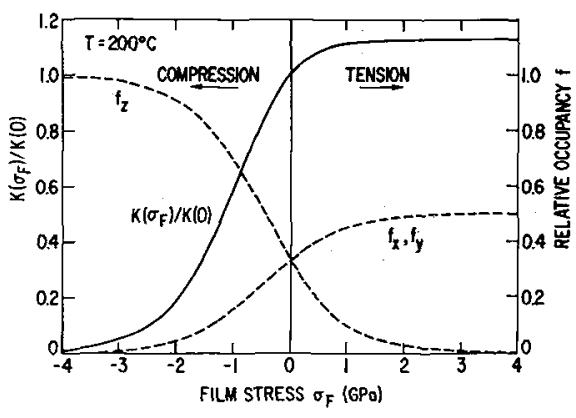

Fig. 3: Calculated results for the effect of film stress on Snoek ordering and relaxation due to oxygen in a $\{100\} \mathrm{Nb}$ film. The broken curves indicate the equilibrium fraction $f$ of interstitials in $\mathrm{X}, \mathrm{Y}$ or $\mathrm{Z}$ sites. The solid curve is the calibration factor $K\left(\sigma_{F}\right)$, relative to its unstressed value $K(0)$.

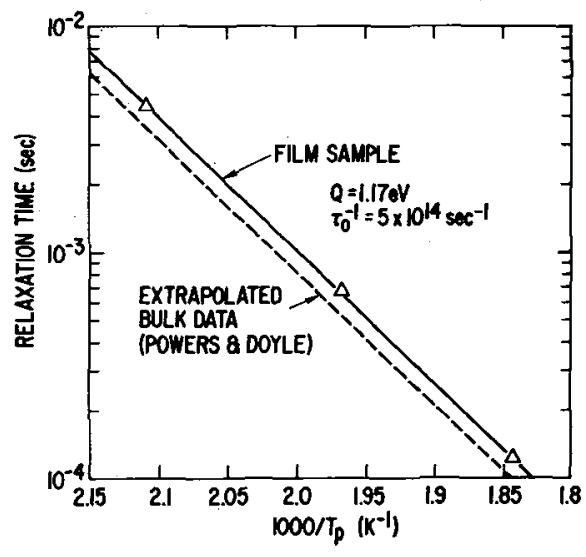

Fig. 4: The Arrhenius plot obtained from the oxygen Snoek peak in supported Nb films, compared with the extrapolation of data obtained from bulk specimens by Powers and Doyle. 
function of $\sigma_{F}$. Interestingly, it is found that if the stress is tensile, this effect is relatively small. Even at the limit where the Z-sites are empty, the ratio $K\left(\sigma_{F}\right) / K(0)$ only changes from 1 to $9 / 8$. On the other hand, the effect of a compressive stress is much more striking. Fig. 3 shows that a compressive stress of $2 \mathrm{GPa}$ would reduce the value of $K$ to only one-fifth of the zero-stress value.

Apart from the effect on $K$, it should also be recognized that the film stress may exert an effect on the kinetics of the Snoek relaxation and the nature of diffusion in the film. For example, if in Fig. 2 the Z-sites become strongly depopulated by a large tensile film stress, diffusion in the film will degenerate from a 3-dimensional to a 1-dimensional process, with the interstitial atoms moving only along linear chains of sites of the type XYXYXY.... While this has a small geometrical effect on the prefactor $\tau_{0}$ governing the relaxation time $\tau$, it is also possible that the stress may have a stronger effect on the kinetics by modifying the activation energy. So far, however, as shown by Fig. 4, a difference of activation energy has not been detected experimentally.

\subsection{Grain-Boundary Sliding in Aluminum Films}

For several years, films of pure aluminum were used to form the metallic interconnections on silicon integrated circuits. More recently, pure aluminum has largely been replaced by various dilute alloys. The reason for the changeover is well known in the microelectronics industry as the 'electromigration problem'- the susceptibility of pure aluminum interconnections to failure, at high current densities and relatively low temperatures, by electrotransport at grain boundaries.[3] These failures, and the investigations they provoked, provided the first clue that the activation energy for grain-boundary diffusion in pure aluminum might be as small as $0.5 \mathrm{eV}$. By contrast, it has been known since the classic work of Kê[4] that the activation energy of the grain-boundary relaxation in bulk polycrystalline aluminum is about $1.4 \mathrm{eV}$, close to the value expected for lattice diffusion. In view of this striking discrepancy, we have employed the vibrating-reed technique to investigate the behavior of aluminum films. The results obtained are of particular interest, since they are the first to demonstrate that the relaxation behavior of films can be strikingly different from that of bulk samples.

Several of the important results which have emerged from an extensive investigation can be illustrated by Fig. 5, which refers to a composite sample consisting of $0.1 \mu \mathrm{m}$ films of pure aluminum evaporated onto a $50 \mu \mathrm{m}$ silica substrate. To start with, we may comment on several experimental points. First, despite the fact that the $t / d$ ratio is now only 0.004 , the substrate damping is again negligibly small. Second, since the thin films do not damp the substrate too heavily, measurements were possible at many tones. The frequencies shown in Fig. 5 correspond to the 1st, 2nd, 4th and 8 th tones, corresponding to a 


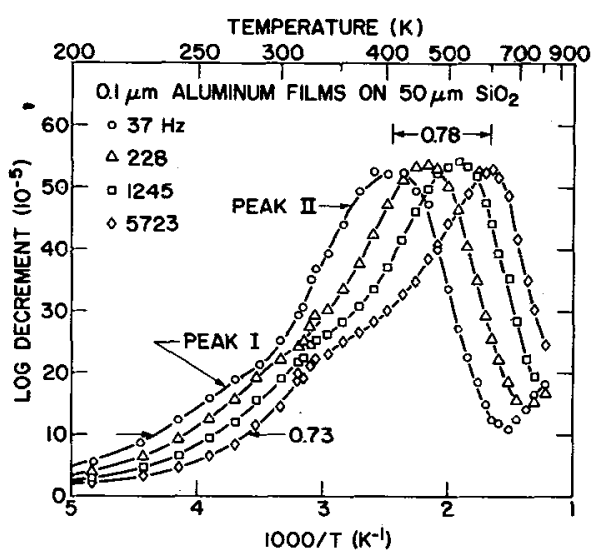

Fig. 5: Internal friction peaks associated with two stages of grain-boundary sliding in supported aluminum films. The sample had been annealed at $550^{\circ} \mathrm{C}$ prior to the measurements. (a)

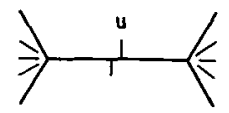

(b)

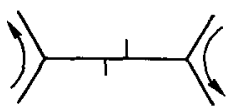

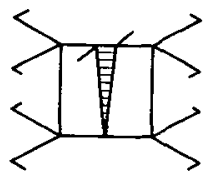

(c)

Fig. 6: Schematic illustration of grainboundary sliding (a) with elastic accommodation, (b) with diffusional accommodation, (c) in a thin film with elastic or diffusional accommodation in the film and pinning to an elastic substrate.

maximum frequency ratio of 154 . Third, since the grain size of the films is essentially identical to the film thickness, the measurements represent the behavior of samples with a grain size that is approximately 3 orders of magnitude smaller than for previous studies of bulk samples. The data reveal two overlapping internal friction peaks, with the smaller peak (I) located below the larger peak (II). As can be appreciated immediately from the near-equality of the $1000 / \mathrm{T}$ intervals marked in Fig. 5 (0.73 and 0.78 ), both peaks have an activation energy of essentially the same mean value. Significantly, this value is $0.55 \mathrm{eV}$, a completely different result from that for the grain-boundary relaxation in bulk samples. Nevertheless, based on a detailed consideration of the peak shapes and magnitudes, and an investigation of the effects of annealing, film thickness, grain size, and alloying additions, we have for reasons given below concluded that both peaks of Fig. 5 are grain-boundary peaks.

Fig. 6(a) shows the picture of grain-boundary relaxation in an idealized polygonal aggregate, as it has been visualized since the work of Zener. Here, grain-boundary sliding, represented by the offset $u$, is accommodated by stress concentrations near the grain corners. These stresses are essential to the recoverable nature of the relaxation in bulk specimens, since they are responsible for the reversal of sliding when the load is removed. In the terminology of Raj and Ashby[5], Zener's model corresponds to grain-boundary sliding with elastic accommodation. Fig. 6(b) shows a continuation of sliding into a second regime, known as sliding with diffusional accommodation. Here the rate of sliding is 
governed by the diffusion of atoms away from regions which sliding forces together, towards regions that tend to separate. Depending on the grain size and temperature, Raj and Ashby have pointed out that this process may be dominated either by grain-boundary diffusion or by lattice diffusion. In normal bulk samples, sliding with diffusional accommodation does not produce a grain-boundary peak because diffusional creep is not recovered on removal of the load. This behavior contributes instead to the high-temperature background damping. In supported thin films, however, the situation is entirely different, since accommodation with the substrate provides an added constraint to grain-boundary sliding. If the substrate behaves elastically, it serves to produce an enforced recoverability of sliding with diffusional accommodation that the detached film would not itself exhibit. We conclude therefore, that elastically-supported thin films may exhibit two regimes of recoverable grain-boundary sliding, namely

I: sliding with elastic accommodation in both the film and substrate and

II: sliding with diffusional accommodation in the film and continued elastic accommodation in the substrate.

These are the two stages of sliding which we associate with peaks I and II in Fig. 5. A schematic picture of the pattern of sliding displacements in the films of Fig. 5 is shown in Fig. 6(c). This diagram is drawn to simulate the grain structure revealed by transmission electron microscopy. The films consist of a single layer of grains, with a grain size about equal to the film thickness, and with the boundaries perpendicular to the substrate. The sliding displacements through the thickness of the film are indicated by the V-shaped shaded area, which is drawn to indicate complete pinning at the interface with the substrate. (Complete pinning need not occur if interfacial sliding is also possible, but this does not appear to be the case in the present experiments).

Based on the explanation of peaks I and II in terms of two stages of grain-boundary sliding, we can draw some important conclusions from the fact that both peaks of Fig. 5 exhibit a mean activation energy close to $0.55 \mathrm{eV}$. This equality neatly demonstrates that the atomic jump governing sliding in stage $I$ is the same jump that governs diffusional accommodation in stage II. This result clearly indicates that both stages involve intrinsic grain-boundary diffusion, and we conclude therefore that the present experiments establish a mean value of $0.55 \mathrm{eV}$ for the activation energy of grain-boundary diffusion in pure aluminum. While this result is consistent with the electromigration behavior of films, it is in striking contrast with the $1.4 \mathrm{eV}$ activation energy observed for the grain-boundary relaxation in bulk aluminum. The implication is that in bulk samples, grain-boundary sliding is not rate-limited by boundary diffusion, but by some slower process, involving lattice diffusion, which occurs at grain-boundary ledges or other obstacles to sliding.[6] There are several possible reasons why such obstacles may not be important in fine-grained 
thin films. First, the much finer grain size favors boundary diffusion as the ratedetermining transport process. Second, the finer grain size may mean that many boundaries do not contain obstacles. Third, the magnitude of the sliding displacement $u$ is also greatly reduced, so there may be less chance of it becoming obstructed. A rough estimate of the sliding displacement is obtained from the approximation

$$
\varepsilon_{a n} \simeq u / g
$$

where $\varepsilon_{a n}$ is the anelastic strain and $g$ is the grain size. Since the strain-amplitude of measurement is about the same in the bulk and the film experiments, $\varepsilon_{a n}$ and $u / g$ are also about the same in both cases. As a consequence of the finer grain size, therefore, the experiments of Fig. 5 represent a situation in which the displacement $u$ is about 3 orders of magnitude smaller than in bulk experiments.

\subsection{Hydrogen-Related Anelasticity in Metallic Glasses}

For our last illustrative example, we have chosen some recent work on metallic glasses. While many of these amorphous alloys have been prepared by thin-film techniques, the majority continue to be prepared by liquid-quenching methods. These methods provide free-standing ribbons or foils of an ideal form for the vibrating-reed technique.

As is well-known, metallic glasses have been the subject of intense research in recent years, and important applications are now on the horizon. Vibrating-reed studies have in the past led to the discovery of reversible magnetic annealing and an unusually large $\Delta$ E-effect in some ferromagnetic metallic glasses.[7,8,9] More recent work has revealed several very interesting anelastic phenomena associated with the presence of hydrogen. These include a short-range reorientation relaxation[10], a long-range Gorsky relaxation[11], and (in the case of ferromagnetic alloys) a magnetoelastic relaxation.[12] We shall deal here only with the first two of these relaxations.

The first example of the hydrogen reorientation peak was discovered in detached sputtered films of nominal atomic composition $\mathrm{Nb}_{75} \mathrm{Ge}_{25}$, although at the time the presence of hydrogen in the films was not known.[13] After the role of hydrogen was established, it was proposed that hydrogen atoms occupy interstitial sites of distinct asymmetry, and that the relaxation involves jumps of hydrogen atoms between sites of different orientations.[14] In view of the generality of this model, we have since looked for the peak in other metallic glasses, and have found it to be a general phenomenon. So far, we have investigated 10 different alloys, including both metal-metalloid and metal-metal glasses, and have produced peaks in all of them by suitable hydrogenation treatments. Important examples include $\mathrm{Pd}_{80} \mathrm{Si}_{20}, \mathrm{Fe}_{80} \mathrm{~B}_{20}$ and $\mathrm{Nb}_{40} \mathrm{Ni}_{60}$. The peaks are located near or below room-temperature, and are large enough to be well-resolved from the background. In 
$\mathrm{Pd}_{80} \mathrm{Si}_{20}$, where the peak can be produced by simple exposure to hydrogen gas, we have found that the peak height varies with pressure $P$ as $P^{1 / 4}$ in contrast to the $P^{1 / 2}$ variation expected if Sievert's law were valid and the peak height varied linearly with concentration. We note in passing that a $P^{1 / 4}$ dependence has also been noted in the hydrogen sorption work of Spit et al.[15]. All the peaks are characterized by a prefactor $\tau_{0}$ of about $10^{-14}$ sec, are about 3 times wider than a Debye peak, and are characteristically asymmetrical when plotted against $1 / \mathrm{T}$. The mean activation energy $\bar{Q}_{R}$ is somewhat concentration dependent. The smallest known value of $\bar{Q}_{R}(0.33 \mathrm{eV})$ occurs in $\mathrm{Pd}_{80} \mathrm{Si}_{20}$; in most of the other alloys $\bar{Q}_{R}$ is in the neighborhood of $0.5 \mathrm{eV}$. A number of very similar peaks have also been observed by Kunzi et al. in a number of metal-metal glasses, including $\mathrm{Cu}_{50} \mathrm{Zr}_{50}[16,17]$. However, those workers found no direct evidence for the involvement of hydrogen and favored the view that the peaks in their metal-metal alloys reflected an intrinsic effect. By contrast, we have recently produced in the glassy alloy $\mathrm{Cu}_{57} \mathrm{Zr}_{43}$ the largest hydrogen peak yet known in a metallic glass. As shown in Fig. 7, the peak is so large that the corresponding modulus defect is easily observed. It appears that the ability of $\mathrm{Cu}-\mathrm{Zr}$ glasses to sorb and desorb hydrogen exhibits an extreme surface sensitivity. Annealing a freshly-cleaned surface at too high a temperature appears to inhibit the sorption or desorption of hydrogen, due presumably to the formation of a surface oxide. Based on present findings, we believe that all of the peaks observed by Künzi et al. are probably hydrogen peaks.

Surface phenomena also play a role in another area of recent progress, which concerns the study of the Gorsky relaxation due to hydrogen in a metallic glass. Measurements of
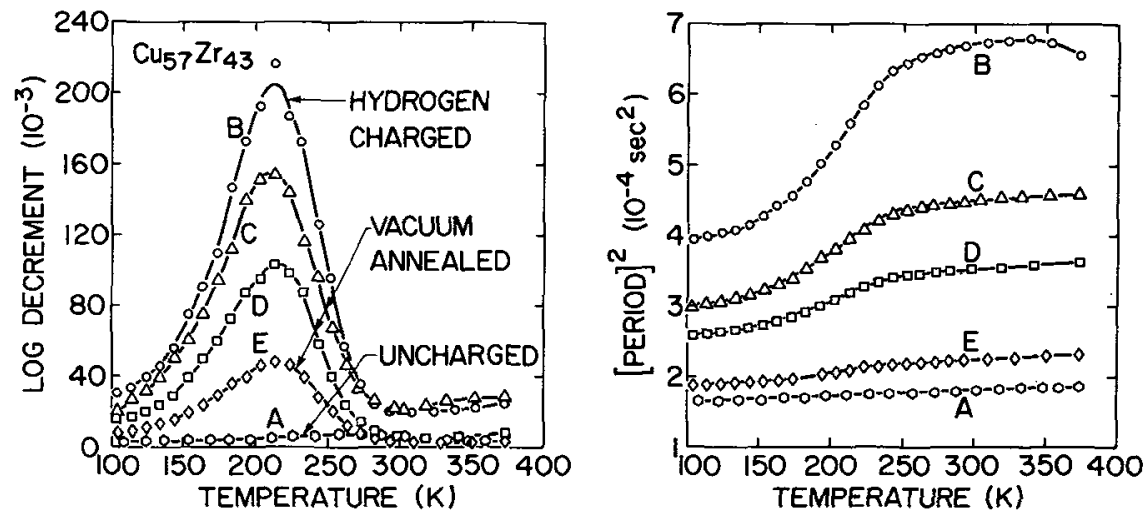

Fig. 7: Response of surface-cleaned amorphous $\mathrm{Cu}_{57} \mathrm{Zr}_{43}$ to hydrogencharging and desorption treatments. A: uncharged; B: after $64 \mathrm{hr}$ in 0.15 $\mathrm{MPa}$ of $\mathrm{H}_{2}$ at $100^{\circ} \mathrm{C}$. Curves $\mathrm{C}, \mathrm{D}$ and $\mathrm{E}$ were obtained after vacuum desorption treatments at $100^{\circ} \mathrm{C}$ for 3,21 and $111 \mathrm{hrs}$, respectively. 
this relaxation in $\mathrm{Pd}_{80} \mathrm{Si}_{20}$ have been greatly facilitated by the slowness of the surfacecontrolled desorption exhibited after electrolytic charging in arsenically-doped sulfuric acid.[10,11] The activation energy for hydrogen diffusion in $\mathrm{Pd}_{80} \mathrm{Si}_{20}$, as derived from the Gorsky relaxation, is $0.25 \mathrm{eV}$. While this is somewhat smaller than $\bar{Q}_{R}$ for the reorientation relaxation, the agreement is close enough to support the idea that reorientation and migration occur by the same family of jumps. This important conclusion supports the view that the reorientation relaxation is basically an analog of the Snoek relaxation, where every interstitial atom serves as an elastic dipole. This in turn leads to the conclusion that hydrogen occupies an interconnected set of low-symmetry interstitial sites in metallic glasses. From a geometrical point of view, we might expect that the sites for hydrogen in a metallic glass are the residue of small holes, inherited from the Bernal structural prototype, that are too small to accommodate glass-forming atoms. However, it remains to be seen whether occupancy of these sites is compatible with the results of the relaxation experiments.

4.Concluding Remarks.- Although the concept of the composite-reed for internal friction studies of thin layers is not new [18, 19], the work described here, coupled with a number of earlier investigations $[20,21,22]$, appears to represent the first successful reduction to practice. What has been learned so far indicates that much further work remains for the future. The most exciting aspect of these studies, as illustrated by the work on aluminum films, is the finding that thin films can provide new behavior and new insights that are not obtainable with bulk samples. Consequently, we believe that thin-layer materials may become a substantial new area for internal friction studies. Finally, turning to metallic glasses, it now appears that anelasticity due to hydrogen is emerging as a general phenomenon, with a number of applications for solubility, dilatational, and kinetic studies. Discoveries of relaxation phenomena that are common to a whole class of materials have been rare in recent years. The Snoek, Zener, grain-boundary and Bordoni relaxations were discovered in the 1940's, and the Hasiguti peaks in the 1950's. The apparent generality of hydrogen-related anelasticity in metallic glasses gives encouragment that major new classes of relaxation phenomena may still await discovery.

Acknowledgment.- Portions of this work were supported in part by the U.S. Army Research Office, Durham.

\section{References}

1. B.S. Berry and W.C. Pritchet, IBM J. Res. Dev. 19, 334 (1975).

2. R.W. Powers and M.V. Doyle, J. Appl. Phys. 30, 514 (1959). 
3. F.M. d'Heurle and P.S. Ho in Thin Films - Interdiffusion and Reactions, edited by J.M. Poate, K.N. Tu and J.W. Mayer, John Wiley and Sons (1978), Chapter 8.

4. T.S. Kê, Phys. Rev. 71, 533 (1947) and 72, 41 (1947).

5. R. Raj and M.F. Ashby, Met. Trans. 2, 1113 (1971).

6. G. Roberts and G.M. Leak in Internal Friction and Ultrasonic Attenuation in Solids -VI, edited by R.R. Hasiguti and N. Mikoshiba, University of Tokyo Press (1977), pp 117-125.

7. B.S. Berry in Metallic Glasses, edited by J.J. Gilman and H.J. Leamy, American Society for Metals, Metals Park, Ohio (1978). Chapter 7.

8. B.S. Berry and W.C. Pritchet, Phys. Rev. Lett. 34, 1022 (1975).

9. B.S. Berry and W.C. Pritchet, AIP Conf. Proc. 34, 292 (1976).

10. B.S. Berry and W.C. Pritchet, Scripta Met. 15, 637 (1981).

11. B.S. Berry and W.C. Pritchet, Phys. Rev. B, to be published.

12. B.S. Berry and W.C. Pritchet, J. Appl. Phys. 52, 1685 (1981).

13. B.S. Berry, W.C. Pritchet and C.C. Tsuei, Phys. Rev. Lett. 41, 410 (1978).

14. B.S. Berry and W.C. Pritchet in Rapidly Quenched Metals III, edited by B. Cantor, The Metals Society, London (1978), Vol. 2, p. 21.

15. F.H.M. Spit, J.W. Drijver and S. Radelaar, Z. Phys. Chem. (Weisbaden) 116, 809 (1979).

16. H.U. Künzi, K. Agyeman and H.-J. Güntherodt, Solid State Commun. 32, 711 (1979).

17. H.U. Künzi, in Liquid and Amorphous Metals, edited by E. Lüscher and H. Coufal, Sijthoff and Noordhoff, Alphen aan den Rijn, The Netherlands, (1980), p. 161.

18. G.P. Weiss and D.O. Smith, Rev. Sci. Inst. 34, 522 (1963).

19. W. Benoit, Helv. Phys. Acta 37, 205 (1964).

20. S.I. Tan, B.S. Berry and W.F.J. Frank, in Ion Implantation in Semiconductors and other Materials, edited by Billy L. Crowder, Plenum, New York (1973) p. 19.

21. B.S. Berry and W.C. Pritchet, in Ion Implantation in Semiconductors 1976, edited by F. Chernow, J.A. Borders and D.K. Brice, Plenum, New York, (1977) p. 435.

22. B.S. Berry and W.C. Pritchet, Thin Solid Films 33, 19 (1976). 